\title{
BASIC INDEXES AND ALUTHGE TRANSFORMATION FOR 2 BY 2 MATRICES
}

\author{
TOSHIYUKI TAKASAKI
}

Abstract. For $2 \times 2$ matrices we provide precise forms of their basic properties such as norms, polar decompositions and singular decompositions. Using these facts, then, we show the exact form of the Aluthge transform $\Delta(A)$ of a matrix $A$ and give an alternative proof of Ando-Yamazaki's result of the convergence of iterations $\left\{\Delta^{n}(A)\right\}$. In case $|\lambda|=|\mu|$ for the eigenvalues $\lambda, \mu$ of $A$ we determine the precise forms of their limits (Theorem 3 ).

Mathematics subject classification (2000): 15A60, 15A18. values.

Key words and phrases: Aluthge transform, polar decompositions, singular decompositions, singular

\section{REFERENCES}

[1] A. ALUTHGE, On $p$-hyponormal operators for $0<p<1$, Integral Equations Operator Theory, 13 (1990), 307-315.

[2] T. ANDO, Aluthge transforms and the convex hull of the eigenvalues of a matrix, Linear Multilinear Algebra, 52 (2004), 281-292.

[3] T. ANDO AND T.YAMAZAKI, The iterated Aluthge transforms of a 2-by-2 matrix converge, Linear Algebra Appl., 375 (2003), 299-309.

[4] T. YAMAZAKI, An expression of spectral radius via Aluthge transformation, Proc. Amer. Math. Soc., 130 (2002), 1131-1137. 